\title{
The mechanism of bronchial asthma. Why do the most serious asthma attacks occur at night?
}

Between 100 and 150 million people around the globe suffer from bronchial asthma and this number is rising. World-wide deaths from this condition have reached over 180,000 annually. The problem is very serious.

\section{KEYWORDS: Arteriovenous anastomoses, cardiovascular diseases, edemata}

\section{Goal}

The author's research is an attempt to establish the new mechanism for asthma. This article is an attempt to study the causes of bronchial asthma and certain cardiovascular disease (CVD) in humans. Why are most of the cardiovascular diseases (CVD) with unknown etiology? This is an attempt to solve the riddle. This is an attempt to study the unknown role of the arteriovenous anastomoses (AVA).

\section{Methods}

Due to new assumptions about the role of abnormal arteriovenous anastomoses (AVA) [1] and the new theory of arrhythmia [2], on which Ermoshkin have been working since 2011, new prospects in unraveling the secrets of cardiovascular disease have opened. He participated in several medical conferences at the People's Friendship University (Moscow) [3], S-Petersburg (Cardiostim-2016) [4], and Brisbane (Arrhythmia-2016) [5] where he had several discussions with the famous cardiologists.

\section{Results}

Many feel that progress in theoretical cardiology has ceased over the last few decades. The mortality caused by cardiovascular diseases in most developed countries is unacceptably high. There is still a lot of unknown or unproven regarding the causes of chronic obstructive pulmonary disease (COPD) and understanding of the causes of bronchial asthma (BA). There are many hypotheses, but they are only partially supported by some experts [68]. Effective treatment still doesn't exist. It is believed that these diseases with an unknown etiology are because the true cause remain unknown.

There are a number of clinical signs of the presence of asthma in humans. But official medical studies consider inflammation of the respiratory tract to be disorders of circadian rhythms in contact with allergens. Progress in this field has ceased for many decades as can be seen in medical practice.

In an effort to address this author developed "New Theory of arrhythmias and cardiovascular disease" in 2011 and continues developing it today. Maybe somebody would pay attention to it? As the author of this new theory, he has seen that the interest in research and hypotheses is increasing every year day by day.

On the basis of the new theory, it turns out that almost all the cardiovascular diseases (CVD) with unknown etiology are caused by anastomoses AVA (maybe groups of AVA), which increase the venous pressure on the system and local levels and block the capillary circulation. Generally speaking, in a healthy person the AVAs can be opened only under significant physical or psychological stress in order to sharply decrease the peak arterial pressure. The main difference between healthy and unhealthy person is a process of closing the AVA, it closes in a healthy person completely and in time.

Let's suppose, furthermore, that in all the organs, portal system, liver, spleen and elsewhere, the hearts power is detrimental to venous pressure. In all the organs, where venous pressure is increased, the heart is "guilty" in causing it! Only the own heart with the use

\section{Vladimir Ermoshkin*}

Russian New University (RosNOU), Moscow, street Radio, 22. Russia

*Author for correspondence: evlad48@list.ru 
of AVA can increase the pressure in veins beyond the norm, and no other more powerful mechanism exists! Apparently, a person can be considered as healthy only until any direct uncontrolled leakage of arterial blood into the veins or intercellular fluid occurs. Otherwise, at least the weight gain and edema appear.

Official medicine mistakenly believes that the venous pressure may increase due to the presence of some "obstacles" such as a blood clot or a tumor. But the "obstacle" is a passive element, it has to increase the pressure in the veins for a short time, it cannot do it for a long time. The pressure increase in veins and their extension occur only due to their direct communication with the arteries. These phenomena are proven using the Cardiocode device [9].

The opening and failure of timely AVA's closure leads to long-term discharge of arterial blood into the veins. Capacitive veins increase their volume and pressure and vena cava is significantly expanded. Depending on the bodies position, the blood may retrogradely enter the venous system of any organ from the vena cava. Mixed blood goes backwards to the small veins and venules trying to stay as low as possible, i.e. it firstly goes to the lower extremities. Edema appear first on legs close to feet, then to increasing heights. Over the years, taking into account the modern lifestyle and hypodynamia, edema cannot "disappear" during sleep in a horizontal position, i.e. during 8 hours.

Over the years, edemata occur in legs (say goodbye a slender legs!), then in the pelvic area (say goodbye to healthy sex!), then in the area of liver and spleen (say goodbye to merry feasts with alcohol!), then ascites appear (say goodbye to brisk walking and jogging!), and finally an increased level of venous pressure reaches the lungs (that is really serious!).

Of course, the sequence of edemata and stagnations varies from person to person, but the following rule remains: the direction is bottomup. The mechanism and sequence of edemata, starting from feet and finishing with lungs, is the same. According to the conventional medicine terminology, most often the right-sided heart failure appears first followed by the left.

It is interesting that both failures sometimes occur in a healthy heart. And it becomes clear due to the new theory.

In this article we do not speak about the circulatory system of the head and conditions of cerebral edema, as there are big differences in hydrostatics. This is a topic for another article. But the mechanism of edemata is the same, they are caused by opened AVAs.

Let me emphasize that lungs have a mixed circulatory system. Vessels of large and small circles of blood are located parallel to each other in the lung tissues. And this feature is a key to understanding the mechanism of bronchial asthma.

When edemata in the lower half of the body occur, the next free volume where the excess venous blood can be placed is the heart, lungs and upper extremities tissue. In the daytime the venous pressure is high in patients with pathologically opened AVAs in a standing or sitting position, but most often it is insufficient for the pulmonary edema formation. But when a patient takes a horizontal position the physical conditions for pulmonary edema may occur.

Due to the opened AVAs the venous blood with high pressure surges into the small venules and veins of the systemic circulation. As the pressure exceeds the threshold values, the fluid penetrates into the intercellular space. In the standing and sitting positions it barely goes to the lungs, as they are located higher, on the same level with the heart, thus all this dirty mixture moves mainly to the legs due to gravity.

But when a person is in a lying position the blood does not move to the legs as while in the horizontal position, the venous pressure is almost everywhere the same causing the venous blood to spread in all directions, both to the head and legs. It can reach the lung tissue and cause edema, the same edema as in all organs below the diaphragm. The mechanism is the same. Then excess intercellular fluid with increased pressure will eventually penetrate into the alveoli, which belong to a small circle of circulation. Therefore the $\mathrm{CO}_{2}$ and $\mathrm{O}_{2}$ exchange is disordered in a person with $\mathrm{BA}$,and he begins to suffocate and choke with phlegm! The phlegm (fluid) is an excessive blood plasma passed through the opened AVAs along large veins to the lungs and then to alveoli.

Recently, one more indirect proof of the plausibility of the author's proposed theory appeared. To know more about testing of the "revolutionary" method of struggle against hypertension read the reports $[10,11]$. The device ROX AV Coupler was used in the testing, it is developed by the American company ROX 
Medical. Tests were carried out in England in 2014-2015. This device is essentially an artificial implant (arteriovenous shunt) with a $4 \mathrm{~mm}$ diameter hole and a continuous throughput of about $800 \mathrm{ml} / \mathrm{min}$. The shunt was placed between the iliac artery and the adjacent vein. Tests were conducted on hypertensive patients resistant to treatment drugs. Due to this method of treatment of resistant forms of hypertension it it successfully showed that a reduction in systemic blood pressure by an average of 27/20 $\mathrm{mm} \mathrm{Hg}$ occurred. Unfortunately, in the 2-9 months over the distal shunt, stagnation of venous blood and edemata appeared in 29\% of cases, which is the same as what occurs in natural permanently opened anastomosis [3,5]. One may ask why arrhythmias occur in the open natural arteriovenous anastomoses [2,3], and not in the permanently opened ROXshunt. The reason is, as stated by researchers, the implant method contributes to treatment of not only arrhythmias, but some other CVDs [12].

In author's opinion, this occurs because the implant is made of solid material, which is significantly different from natural tissue of anastomoses, so the mechanical waves traveling along the walls of arteries are effectively reflected from foreign material and do not hit the vena cava wall. Thus, ROX Coupler reliably eliminates the cause of arrhythmias due to the impact of pulse waves. The ROX Coupler implant, struggling against the effects of hypertension, reduces the blood pressure and thereby eliminates the reason for the opening and closing of natural AVA. We can assume that after the installation of ROXshunt the large natural AVA are forced to be in the closed state permanently, and the heart, lungs and some large vessels have to work with the increased load. It is useless to pump some extra volume of blood from major arteries into the veins. This excess value of pumping is 800 $\mathrm{ml} / \mathrm{min}$ or $16 \%$ of the norm in $5000 \mathrm{ml} / \mathrm{min}$. In author's opinion, this solution is not optimal. It is obvious that for hypertensive patient in the future it is needed to develop the implants with a variable diameter of shunt lumen, down to zero value of lumen, depending on the current blood pressure in the arteries. But it is still better to "use" the natural anastomoses given to us by nature, and for that it is necessary to lead a healthy lifestyle with daily moderate physical loads on muscles and cardiovascular system. Another confirmation of the fact that the mechanical impulses are suppressed heterogeneity of tissue or a surgical suture is statistics on the results of greater efficiency bicaval (compared to biatrial) technique in heart transplant [13].

\section{Conclusions}

1. This theory should be still seen as a promising hypothesis, because author's research is not generally accepted yet. The author believes and hopes that the cause of many cardiovascular diseases with unknown etiology becomes clearer. At least the cause of heart premature beats and paroxysmal tachycardias looks quite reasonable in the framework of author's new theory.

2. In accordance with the proposed new theory the methods of diagnostics and treatment of bronchial asthma and some other lung diseases should be reviewed.

3. A key element of the forthcoming research and experiments is a study of performance of the large arteriovenous anastomoses (AVAs) located mainly near the liver, portal and hepatic veins, liver and mesenteric arteries, vena cava. The key to treatment is apparently a thin adjustment of the AVAs performance, detection and tracking of the retrograde venous blood movement.

4. It is natural that physical activity throughout the day, adequate sleeping time, special breathing exercises for involving the stagnant venous blood into the systemic circulation may be advised to prevent the cardiovascular disease.

5. The author is ready to cooperate with all interested parties.

\section{REFERENCES}

Ermoshkin V. Las Vegas, USA. Arteriovenous anastomoses and cardiovascular diseases. $8^{\text {th }}$ Cardiovascular Nursing \& Nurse Practitioners Meeting. (2016).

Ermoshkin V. New theory of arrhythmia. Conceptual substantiation of arrhythmia mechanisms. Cardiometry (8), 6-17 (2016).

Ermoshkin V. "A New Theory of arrhythmia," clarifies the causes of arrhythmias and some "metabolic disorders». Journal of «Health and Education in the 21st Century.» 17(9), 1-9
(2015).

Ermoshkin V. Cardiostim. New hypothesis of cardiac arrhythmias in humans.

Ermoshkin V. Australia. Brisbane. Arrhythmia. Proceedings of the International Conference on arrhythmia (2016).

Bethesda. Expert Panel Report 3: Guidelines for the Diagnosis and Management of Asthma. National Heart, Lung, and Blood Institute (US). (2007).

Cohn L, Elias JA, Chupp GL. Asthma: mechanisms of disease persistence and progression. Annu Rev Immunol. 22, 789-815 (2004).

Bethesda Expert Panel Report: Guidelines for the Diagnosis and Management of Asthma. National Heart, Lung, and Blood Institute (US). (2002).

Lukyanchenko V. Cardiometric signs of performance of arteriovenous anastomosis in human cardiovascular system. Cardiometry (8), 22-25 (2016).

Lobo MD, Sobotka PA, Stanton A, et al. Central arteriovenous anastomosis for the treatment of 
patients with uncontrolled hypertension (the ROX CONTROL HTN study): a randomised controlled trial. The Lancet. 385(9978), 16341641 (2015).

Kapil, Vikas, Paul A. Sobotka, et al. "Central Iliac
Arteriovenous Anastomosis For Hypertension: Targeting Mechanical Aspects Of The Circulation", Curr Hypertens Rep. 17(9), 73 (2015).

Rodney B. Methods, systems and devices for treating cardiac arrhythmias, Applicaton: \#20160058452. Freshpatents. (2016)

Ermoshkin V. Heart transplantation mysteriously eliminates arrhythmia. Cardiometry. (8), 18-21 (2016). 